\title{
Numerical study on the performance of nozzle flow for supersonic chemical oxygen-iodine lasers
}

\author{
Zongmin Hu · Junming Lü · Zonglin Jiang • \\ Rho-Shin Myong · Tae-Hwan Cho
}

Received: 14 September 2006 / Revised: 27 September 2007 / Accepted: 23 November 2007 / Published online: 13 March 2008

(c) Springer-Verlag 2008

\begin{abstract}
Laser performance is greatly dependent on its operating conditions due to the strong coupling among multiphysics such as gas-dynamics, chemical reaction kinetics and optics in the mixing nozzle of COIL. In this paper, 3D CFD technology is used to simulate the mixing and reactive flow of subsonic cross jet scheme at different conditions. Results obtained show that the jet penetration depth plays a dominant role in the spatial distribution of small signal gains. In the case of over-penetration, unsteady flow structures are induced by impinging between the opposing jets. The optimum spatial distribution of the chemical performance cannot be obtained even if the full penetration condition is achieved through the subsonic transverse jet mixing scheme in the COIL nozzle flow.
\end{abstract}

Keywords Chemical oxygen-iodine laser (COIL) .

Numerical simulation - Small signal gains .

Unsteady structures

\section{Introduction}

The chemical oxygen-iodine laser, or COIL, is a high power chemical laser with a short wavelength, and can be operated on an atomic iodine laser transition. The ground state iodine

Z. Hu $\cdot$ J. Lü $\cdot$ Z. Jiang $(\varangle)$

LHD, Institute of Mechanics,

CAS, Beijing 100080, China

e-mail: zljiang@imech.ac.cn

Z. Hu · R.-S. Myong · T.-H. Cho

Research Center for Aircraft Parts Technology,

Geyongsang National University,

Jinju 660-701, South Korea

e-mail: bighumin@126.com atom $\mathrm{I}$, is pumped into an excited state $\mathrm{I}^{*}$, through the process of near resonant energy transfer between meta-steady singlet oxygen $\mathrm{O}_{2}\left({ }^{1} \Delta\right)$, and $\mathrm{I}$. Then, the laser medium, $\mathrm{I}^{*}$ radiates a photon with a wavelength $\lambda=1.315 \mu \mathrm{m}$, which can amplify the seed beam into a high power infrared laser beam through the resonance cavity. The research on the chemical oxygeniodine lasers (COIL) has recently been accelerated due to the potential military and industrial applications, which are dedicated to systematically integrating the COIL for mobile platforms. In order to meet the requirement of such the platform, the enormous volume and weight of the COIL must be reduced significantly. This appears to be difficult task, but it is an interesting research topic.

Although the supersonic COILs are routinely operated in several laboratories around the world and various impressive projects are underway, there still remains room for considerable improvement of the COIL's main performance characteristics [1]. Generally, the laser performance greatly depends on operating conditions due to the strong interactions among multi-physics, such as gas-dynamics, chemical reaction kinetics, and optics in the mixing nozzle flow of the COIL. The recent research indicate that the unsteadiness in the jet mixing region translates directly into unsteadiness in the laser gain and power output [2].

While large numbers of the numerical researches [1-5] regarding the mixing nozzle flow can be found in the literature published in the last decade, so far, few efforts have been made and less attention has been paid to the interaction of the flow structures with the chemical reactions associated with different operating conditions. In this paper, nozzle flows are analyzed further by using 3D CFD technologies and high performance parallel computing resources. The objective of this work aims at obtaining a better understanding of the physical processes in the COIL mixing nozzle operating under different conditions. 


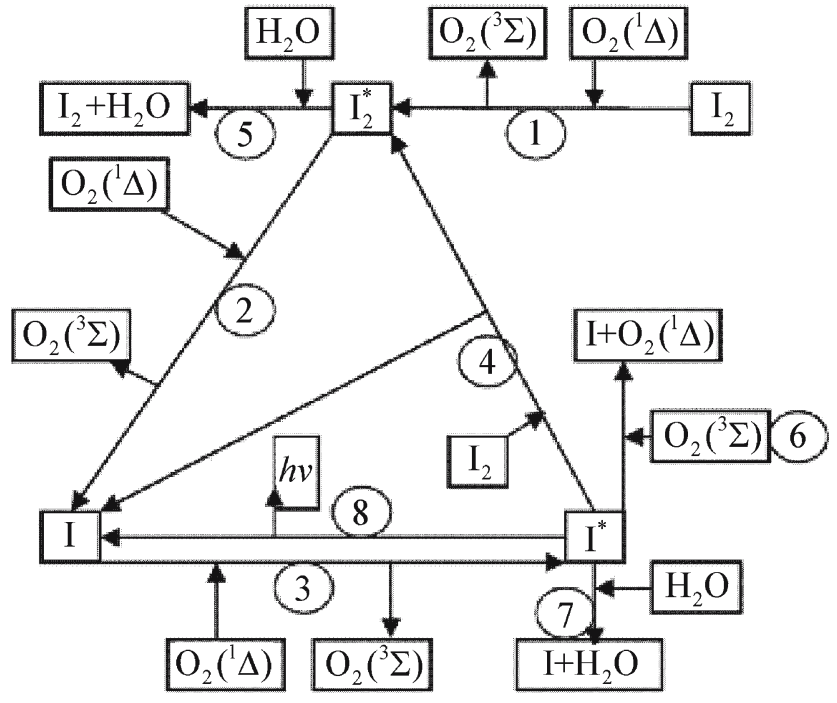

Fig. 1 Sketch of the chain reaction of the dissociation of $\mathrm{I}_{2}$

\section{Chemical reactions and gas dynamics}

In general, the gas phase chemistry in the mixing nozzle of COIL can be described with the following three processes: the pumping reaction produces an excited iodine atom, and various competing or de-active reactions. Although some uncertainties exist in the applicable kinetics rate equations and corresponding rates, predicting the dissociation of molecular iodine $\mathrm{I}_{2}$ in an excited oxygen stream $\mathrm{O}_{2}\left({ }^{1} \Delta\right)$ is the largest source of the errors in modelling the performance of chemical oxygen-iodine lasers [4,5]. The main kinetics processes are illustrated in Fig. 1. Reaction equations (1)-(4) model the self-catalyzed chain reaction of the $\mathrm{I}_{2}$ dissociation, where Eq. (1) is the primary onset reaction and reaction (3) is the resonant energy transfer or pumping reaction through which the laser media are generated. Reaction equations (5)(7) govern different quench processes by third-bodies. Equation (8) represents the stimulated laser radiation. For simplicity, other reaction equations, such as the minor onset reactions of the dissociation, are not included in Fig. 1 but can be found in [6] or in Table 1.

\section{Numerical analysis of the flowfield in COIL}

\subsection{Governing equations and numerical technology}

The COIL flow with a low density and Reynolds number from $10^{2}$ (based on jet flow conditions and the orifice) to $10^{3}$ (based on the primary flow conditions and the inlet chamber size) is governed by 3D Navier-Stokes equations. In Cartesian coordinates, the conservative form of the equations is written as:

$\frac{\partial \boldsymbol{U}}{\partial t}+\frac{\partial \boldsymbol{F}_{i}}{\partial x_{i}}=\frac{\partial \boldsymbol{D}_{i}}{\partial x_{i}}+\boldsymbol{S}_{c} \quad(i=1,2,3)$, where, $\boldsymbol{U}$ is the unknown variable vector, $\boldsymbol{F}_{i}$ are the inviscid flux vectors in $x, y$, and $z$ directions, $\boldsymbol{D}_{i}$ are the diffusion flux vectors, and $\boldsymbol{S}_{c}$ is the chemical reaction source term, where

$$
\begin{aligned}
\boldsymbol{U}= & {\left[\rho_{1} \ldots \rho_{n s}, \rho u_{1}, \rho u_{2}, \rho u_{3}, \rho e\right], } \\
\boldsymbol{F}_{i}= & {\left[\rho_{1} u_{i} \ldots \rho_{n s} u_{i}, \rho u_{i} u_{1}+p \delta_{i 1}, \rho u_{i} u_{2}\right.} \\
& \left.+p \delta_{i 2}, \rho u_{i} u_{3}+p \delta_{i 3},(\rho e+p) u_{i}\right], \\
\boldsymbol{D}_{i}= & {\left[\rho_{1} v_{1 i} \ldots \rho_{n s} v_{n s i}, \tau_{1 i}, \tau_{2 i}, \tau_{3 i}, u_{j} \tau_{i j}+q_{i}\right], } \\
\boldsymbol{S}_{c}= & {\left[\dot{\omega}_{1} \ldots \dot{\omega}_{n s}, 0,0,0,0\right] . }
\end{aligned}
$$

In above equations, the total density of the mixture $\rho=$ $\sum_{\mathrm{sp}=1}^{n s} \rho_{\mathrm{sp}}$, and the partial density of the species sp is denoted by $\rho_{\mathrm{sp}} ; u_{i}(i=1,2,3)$ are velocity components in $x, y$ and $z$ directions; $e$ and $p$ denote the total energy, and the pressure of the mixture; $v_{\mathrm{sp}, i}$ represents the diffusion velocity of the species sp in direction $x_{i} ; \tau$ and $q$ define the viscous stress and heat conduction terms, and $\dot{\omega}_{\mathrm{sp}}$ is the variation rate of the species sp. The transport properties of each pure component, such as viscosity and thermal conductivity, and special heat capacity and enthalpy, are obtained from the relations given in the power series form of temperature. The effective binary diffusion model rather than Fick's model is applied to approximate the diffusion of all species. In the chemical kinetic model, 21 elementary reactions and ten species, $\mathrm{I}_{2}, \mathrm{I}_{2}^{*}$, I, $\mathrm{I}^{*}, \mathrm{O}_{2}\left({ }^{1} \Delta\right), \mathrm{O}_{2}\left({ }^{1} \Sigma\right), \mathrm{O}_{2}\left({ }^{3} \Sigma\right), \mathrm{H}_{2} \mathrm{O}, \mathrm{Cl}_{2}$ and $\mathrm{He}$ are taken into consideration. The transport property model, thermodynamic parameter variations, diffusion model and chemical reactions were synthetically analyzed and presented in [7].

The total energy density is defined as

$\rho e=\rho h-p+\rho u_{i} u_{i} \quad(i=1,2,3)$.

Each species is usually assumed to be a thermally perfect gas, and the specific heat, enthalpy and entropy for each species can be calculated by the thermal polynomial equations

$$
\begin{aligned}
\frac{C p_{i}}{R_{i}}= & a_{1 i} T^{-2}+a_{2 i} T^{-1}+a_{3 i}+a_{4 i} T+a_{5 i} T^{2} \\
& +a_{6 i} T^{3}+a_{7 i} T^{4}+a_{8 i} T^{-3}, \\
\frac{h_{i}}{R_{i} T}= & a_{1 i} T^{-2}+a_{2 i} T^{-1} \ln T+a_{3 i}+\frac{a_{4 i}}{2} T+\frac{a_{5 i}}{3} T^{2} \\
& +\frac{a_{6 i}}{4} T^{3}+\frac{a_{7 i}}{5} T^{4}-\frac{a_{8 i}}{2} T^{-3}+a_{9 i} T^{-1}, \\
\frac{S_{i}}{R_{i}}=- & \frac{a_{1 i}}{2} T^{-2}-a_{2 i} T^{-1}+a_{3 i} \ln T+a_{4 i} T+\frac{a_{5 i}}{2} T^{2} \\
& +\frac{a_{6 i}}{3} T^{3}+\frac{a_{7 i}}{4} T^{4}+\frac{a_{8 i}}{3} T^{-3}+a_{10 i} .
\end{aligned}
$$


Table 1 Elementary reaction set

\begin{tabular}{|c|c|c|c|c|c|c|c|c|}
\hline No. & & & & Reaction & & & & $k_{f}\left(\mathrm{~cm}^{3}\right.$ molecule $\left.^{-1} \mathrm{~s}^{-1}\right)$ \\
\hline 1 & $\mathrm{O}_{2}\left({ }^{1} \Delta\right)$ & + & $\mathrm{O}_{2}\left({ }^{1} \Delta\right)$ & $\rightarrow$ & $\mathrm{O}_{2}\left({ }^{1} \Sigma\right)$ & + & $\mathrm{O}_{2}\left({ }^{3} \Sigma\right)$ & $2.7 \times 10^{-17}$ \\
\hline 2 & $\mathrm{O}_{2}\left({ }^{1} \Sigma\right)$ & + & $\mathrm{H}_{2} \mathrm{O}$ & $\rightarrow$ & $\mathrm{O}_{2}\left({ }^{1} \Delta\right)$ & + & $\mathrm{H}_{2} \mathrm{O}$ & $6.7 \times 10^{-12}$ \\
\hline 3 & $\mathrm{O}_{2}\left({ }^{1} \Delta\right)$ & + & $\mathrm{O}_{2}\left({ }^{3} \Sigma\right)$ & $\rightarrow$ & $\mathrm{O}_{2}\left({ }^{3} \Sigma\right)$ & + & $\mathrm{O}_{2}\left({ }^{3} \Sigma\right)$ & $1.6 \times 10^{-18}$ \\
\hline 4 & $\mathrm{O}_{2}\left({ }^{1} \Delta\right)$ & + & $\mathrm{H}_{2} \mathrm{O}$ & $\rightarrow$ & $\mathrm{O}_{2}\left({ }^{3} \Sigma\right)$ & + & $\mathrm{H}_{2} \mathrm{O}$ & $4.0 \times 10^{-18}$ \\
\hline 5 & $\mathrm{O}_{2}\left({ }^{1} \Delta\right)$ & + & $\mathrm{Cl}_{2}$ & $\rightarrow$ & $\mathrm{O}_{2}\left({ }^{3} \Sigma\right)$ & + & $\mathrm{Cl}_{2}$ & $6.0 \times 10^{-18}$ \\
\hline 6 & $\mathrm{O}_{2}\left({ }^{1} \Delta\right)$ & + & $\mathrm{He}$ & $\rightarrow$ & $\mathrm{O}_{2}\left({ }^{3} \Sigma\right)$ & + & $\mathrm{He}$ & $8.0 \times 10^{-21}$ \\
\hline 7 & $\mathrm{I}_{2}$ & + & $\mathrm{O}_{2}\left({ }^{1} \Sigma\right)$ & $\rightarrow$ & $2 \mathrm{I}$ & + & $\mathrm{O}_{2}\left({ }^{3} \Sigma\right)$ & $4.0 \times 10^{-12}$ \\
\hline 8 & $\mathrm{I}_{2}$ & + & $\mathrm{O}_{2}\left({ }^{1} \Sigma\right)$ & $\rightarrow$ & $\mathrm{I}_{2}$ & + & $\mathrm{O}_{2}\left({ }^{3} \Sigma\right)$ & $1.6 \times 10^{-11}$ \\
\hline 9 & $\mathrm{I}_{2}$ & + & $\mathrm{O}_{2}\left({ }^{1} \Delta\right)$ & $\rightarrow$ & $\mathrm{I}_{2}^{*}$ & + & $\mathrm{O}_{2}\left({ }^{3} \Sigma\right)$ & $7.0 \times 10^{-15}$ \\
\hline 10 & $\mathrm{I}_{2}$ & + & $\mathrm{I}^{*}$ & $\rightarrow$ & I & + & $\mathrm{I}_{2}^{*}$ & $3.5 \times 10^{-11}$ \\
\hline 11 & $I_{2}^{*}$ & + & $\mathrm{O}_{2}\left({ }^{1} \Delta\right)$ & $\rightarrow$ & $2 \mathrm{I}$ & + & $\mathrm{O}_{2}\left({ }^{3} \Sigma\right)$ & $3.0 \times 10^{-10}$ \\
\hline 12 & $\mathrm{I}_{2}^{*}$ & + & $\mathrm{O}_{2}\left({ }^{3} \Sigma\right)$ & $\rightarrow$ & $\mathrm{I}_{2}$ & + & $\mathrm{O}_{2}\left({ }^{3} \Sigma\right)$ & $4.9 \times 10^{-12}$ \\
\hline 13 & $\mathrm{I}_{2}^{*}$ & + & $\mathrm{H}_{2} \mathrm{O}$ & $\rightarrow$ & $\mathrm{I}_{2}$ & + & $\mathrm{H}_{2} \mathrm{O}$ & $1.7 \times 10^{-11}$ \\
\hline 14 & $I_{2}^{*}$ & + & $\mathrm{He}$ & $\rightarrow$ & $\mathrm{I}_{2}$ & + & $\mathrm{He}$ & $9.8 \times 10^{-12}$ \\
\hline 15 & I & + & $\mathrm{O}_{2}\left({ }^{1} \Delta\right)$ & $\rightarrow$ & $I^{*}$ & + & $\mathrm{O}_{2}\left({ }^{3} \Sigma\right)$ & $7.8 \times 10^{-11}$ \\
\hline 16 & $I^{*}$ & + & $\mathrm{O}_{2}\left({ }^{3} \Sigma\right)$ & $\rightarrow$ & I & + & $\mathrm{O}_{2}\left({ }^{1} \Delta\right)$ & $1.028 \times 10^{-10} \mathrm{e}^{-401.4 / \mathrm{T}}$ \\
\hline 17 & I & + & $\mathrm{O}_{2}\left({ }^{1} \Delta\right)$ & $\rightarrow$ & I & + & $\mathrm{O}_{2}\left({ }^{3} \Sigma\right)$ & $1.0 \times 10^{-15}$ \\
\hline 18 & $I^{*}$ & + & $\mathrm{O}_{2}\left({ }^{1} \Delta\right)$ & $\rightarrow$ & I & + & $\mathrm{O}_{2}\left({ }^{1} \Sigma\right)$ & $1.0 \times 10^{-13}$ \\
\hline 19 & $I^{*}$ & + & $\mathrm{O}_{2}\left({ }^{1} \Delta\right)$ & $\rightarrow$ & I & + & $\mathrm{O}_{2}\left({ }^{1} \Delta\right)$ & $1.1 \times 10^{-13}$ \\
\hline 20 & I & + & $I^{*}$ & $\rightarrow$ & I & + & I & $1.7 \times 10^{-13}$ \\
\hline 21 & $I^{*}$ & + & $\mathrm{H}_{2} \mathrm{O}$ & $\rightarrow$ & I & + & $\mathrm{H}_{2} \mathrm{O}$ & $2.1 \times 10^{-12}$ \\
\hline
\end{tabular}

Therefore, the enthalpy of the mixture $h$ is calculated by summing the enthalpies of all species

$h=\sum_{i=1}^{n s} c_{i} h_{i}$,

where $c_{i}$ is the mass fraction of species $i$ in the mixture. According to Dalton's law, the pressure $p$ is the sum of the partial pressures for each species and can be calculated with the state equation for the perfect gas

$p=\sum_{i=1}^{n s} \rho_{i} R_{i} T$

where, $R_{i}$ is the gas constant of species $i$ and $T$ is the temperature of the gas mixture.

For the transport properties, the coefficients of thermal conductivity and the viscosity of the pure species can be approximated by using the following equations.

$\kappa=a+b T+c T^{2}+d T^{3}$,

$\mu=A+B T+C T^{2}$.

The appropriate constants in above two equations can be found in the relevant literature [7]. Also, the transport parameters, $\eta(\kappa, \mu)$, of the mixture are given by Wilkes' equations $\eta_{\text {mix }}=\sum_{i=1}^{n s} \frac{X_{i} \eta_{i}}{\sum_{j=1}^{n s} X_{j} \phi_{i j}}$

$$
\phi_{i j}=\left[1+\left(\frac{\eta_{i}}{\eta_{j}}\right)^{0.5}\left(\frac{w_{j}}{w_{i}}\right)^{0.25}\right]^{2} /\left[8\left(1+\frac{w_{i}}{w_{j}}\right)\right]^{0.5},
$$

where the molecular weight and the molar fraction of each species are denoted by $w_{i}$ and $X_{i}$, respectively.

An accurate description of the molecular diffusion of the chemical species is important for the low pressure flowfields of chemical lasers. The Ramshaw-Dukowicz approximation for multi-component diffusive transport is commonly used, including both concentration gradient driven and pressure gradient driven contributions

$\rho_{i} v_{i}=-\gamma_{t}\left[G_{i}^{\chi}-\frac{\rho_{i}}{\rho} \sum_{j=1}^{n s} G_{j}^{\chi}\right]-\gamma_{t}\left[G_{i}^{p}-\frac{\rho_{i}}{\rho} \sum_{j=1}^{n s} G_{j}^{p}\right]$,

$G_{i}^{\chi}=w_{i} D_{i} \nabla \chi_{i}$,

$G_{i}^{p}=w_{i} D_{i}\left(\chi_{i}-\rho_{i} / \rho\right) \nabla p / p$, 
where $\chi_{i}$ and $\gamma_{t}$ denote the molar concentration of species and the total molar concentration of the mixture, respectively. The average diffusion coefficient $D_{i}$ is calculated by using a binary effective diffusion model.

The production rate resulting from chemical reaction of species $i$ is determined by

$\dot{\omega}_{i}=w_{i} \sum_{r=1}^{N R}\left(v_{i r}^{\prime}-v_{i r}^{\prime \prime}\right)\left(k_{f, r} \prod_{j=1}^{n s}\left(\chi_{j}\right)^{v_{j r}^{\prime}}-k_{b, r} \prod_{j=1}^{n s}\left(\chi_{j}\right)^{v_{j r}^{\prime \prime}}\right)$.

The forward reaction rate constant of each reaction is calculated by using the Arrhenius equation and the corresponding backward reaction rate constant can be derived from the equilibrium coefficient and the forward rate constants

$k_{f, r}=C_{r} T^{n r} \exp \left(-E a_{r} / R T\right)$,

$k_{b, r}=k_{f, r} / k_{e, r}$

$k_{e, r}=\exp \left(\sum_{i=1}^{n s}\left[\left(v_{i r}^{\prime \prime}-v_{i r}^{\prime}\right)\left(\frac{S_{i r}}{R_{i r}}-\frac{h_{i r}}{R_{i r} T}\right)\right]\right) k_{p, r}$,

$k_{p, r}=\left(\frac{P_{\mathrm{atm}}}{R T}\right)^{\sum_{i=1}^{n s}\left(v_{i r}^{\prime \prime}-v_{i r}^{\prime}\right)}$.

In these equations, $i r$ and $j r$ denote the species numbers in the $r$ th elementary reaction; $v_{i r}^{\prime}$ and $v_{i r}^{\prime \prime}$ are the stoichiometric coefficient of species $i$ in the $r$ th elementary reaction as the reactant and product, respectively; $k_{f, r}, k_{b, r}$ and $k_{e, r}$ denote the forward and the backward reaction rate constants, and the equilibrium coefficient of the $r$ th elementary reaction, respectively. The pre-exponential factor $C_{r}$, the temperature index $n r$, and the activation energy $E a_{r}$ are given by the detailed finite rate chemical reaction model.

The semi-discretized difference equation for Eq. (1) is

$$
\left(\frac{\partial U}{\partial t}\right)=-\mathrm{CONV}_{i, j, k}^{n}+\operatorname{VISC}_{i, j, k}^{n}+S_{i, j, k}^{n} .
$$

The dissipative terms VISC are discretized with a second order centered difference scheme, while the convective term CONV, is discretized by using the dispersion controlled dissipative (DCD) scheme proposed by Jiang et al. [8,9] for shock wave capturing, which was developed originally to simulate 2D chemical reactive flows of detonation waves [10] and 3D COIL flows [3]. In a curvilinear 3D space, the semidiscretized form of the convective term is

$$
\begin{aligned}
\operatorname{CONV}_{i, j, k}^{n}= & \frac{1}{\Delta \xi}\left(\hat{F}_{i+\frac{1}{2}}^{n}-\hat{F}_{i-\frac{1}{2}}^{n}\right) \\
& +\frac{1}{\Delta \eta}\left(\hat{G}_{j+\frac{1}{2}}^{n}-\hat{G}_{j-\frac{1}{2}}^{n}\right) \\
& +\frac{1}{\Delta \zeta}\left(\hat{H}_{k+\frac{1}{2}}^{n}-\hat{H}_{k-\frac{1}{2}}^{n}\right) .
\end{aligned}
$$

Here $\hat{F}, \hat{G}, \hat{H}$ are the numerical flux vectors, and

$$
\begin{aligned}
& \left\{\begin{array}{l}
\hat{F}_{i \pm \frac{1}{2}}^{n}=\tilde{F}_{i \pm \frac{1}{2}, j, k}^{+}+\tilde{F}_{i \pm \frac{1}{2}, j, k}^{-}, \\
\hat{G}_{j \pm \frac{1}{2}}^{n}=\tilde{G}_{i, j \pm \frac{1}{2}, k}^{+}+\tilde{G}_{i, j \pm \frac{1}{2}, k}^{-}, \\
\hat{H}_{k \pm \frac{1}{2}}^{n}=\tilde{H}_{i, j, k \pm \frac{1}{2}}^{+}+\tilde{H}_{i, j, k \pm \frac{1}{2}}^{-},
\end{array}\right. \\
& \left\{\begin{array}{l}
\tilde{F}_{i+\frac{1}{2}, j, k}^{+}=\tilde{F}_{i, j, k}^{+} \\
\quad+\frac{1}{2} \Phi_{A}^{+} \operatorname{minmod}\left(\Delta \tilde{F}_{i-\frac{1}{2}, j, k}^{+}, \Delta \tilde{F}_{i+\frac{1}{2}, j, k}^{+}\right), \\
\tilde{G}_{i, j+\frac{1}{2}, k}^{+}=\tilde{G}_{i, j, k}^{+} \\
\quad+\frac{1}{2} \Phi_{B}^{+} \operatorname{minmod}\left(\Delta \tilde{G}_{i, j-\frac{1}{2}, k}^{+}, \Delta \tilde{G}_{i, j+\frac{1}{2}, k}^{+}\right), \\
\tilde{H}_{i, j, k+\frac{1}{2}}^{+}=\tilde{H}_{i, j, k}^{+} \\
\quad+\frac{1}{2} \Phi_{C}^{+} \operatorname{minmod}\left(\Delta \tilde{H}_{i, j, k-\frac{1}{2}}^{+}, \Delta \tilde{H}_{i, j, k+\frac{1}{2}}^{+}\right)
\end{array}\right. \\
& \left\{\begin{array}{l}
\tilde{F}_{i+\frac{1}{2}, j, k}^{-}=\tilde{F}_{i, j, k}^{-} \\
\quad-\frac{1}{2} \Phi_{A}^{-} \operatorname{minmod}\left(\Delta \tilde{F}_{i+\frac{1}{2}, j, k}^{-}, \Delta \tilde{F}_{i+\frac{3}{2}, j, k}^{-}\right), \\
\tilde{G}_{i, j+\frac{1}{2}, k}^{-}=\tilde{G}_{i, j, k}^{-} \\
\quad-\frac{1}{2} \Phi_{B}^{-} \operatorname{minmod}\left(\Delta \tilde{G}_{i, j+\frac{1}{2}, k}^{-}, \Delta \tilde{G}_{i, j+\frac{3}{2}, k}^{-}\right) \\
\tilde{H}_{i, j, k+\frac{1}{2}}^{-}=\tilde{H}_{i, j, k}^{-} \\
\quad-\frac{1}{2} \Phi_{C}^{-} \operatorname{minmod}\left(\Delta \tilde{H}_{i, j, k+\frac{1}{2}}^{-}, \Delta \tilde{H}_{i, j, k+\frac{3}{2}}^{-}\right)
\end{array}\right. \\
& \left\{\begin{array}{l}
\Delta \tilde{F}_{i+\frac{1}{2}, j, k}^{ \pm}=\tilde{F}_{i+1, j, k}^{ \pm}-\tilde{F}_{i, j, k}^{ \pm}, \\
\Delta \tilde{G}_{i+\frac{1}{2}, j, k}^{ \pm}=\tilde{G}_{i, j+1, k}^{ \pm}-\tilde{G}_{i, j, k}^{ \pm}, \\
\Delta \tilde{H}_{i+\frac{1}{2}, j, k}^{ \pm}=\tilde{H}_{i, j, k+1}^{ \pm}-\tilde{H}_{i, j, k}^{ \pm},
\end{array}\right. \\
& \left\{\begin{array}{l}
\tilde{F}^{ \pm}=\tilde{A}^{ \pm} \tilde{U}, \\
\tilde{G}^{ \pm}=\tilde{B}^{ \pm} \tilde{U}, \\
\tilde{H}^{ \pm}=\tilde{C}^{ \pm} \tilde{U},
\end{array}\right. \\
& \left\{\begin{array}{l}
\Phi_{A}^{ \pm}=I \mp \beta \Lambda_{A}^{ \pm}, \\
\Phi_{B}^{ \pm}=I \mp \beta \Lambda_{B}^{ \pm}, \\
\Phi_{C}^{ \pm}=I \mp \beta \Lambda_{C}^{ \pm},
\end{array}\right. \\
& \beta=\Delta t / \delta r, \quad r=\xi, \eta, \zeta .
\end{aligned}
$$

In the above formulas, $\tilde{F}, \tilde{G}$ and $\tilde{H}$ are flux vectors in the curvilinear coordinate directions $\xi, \eta, \zeta$, respectively; $\tilde{A}=\partial \tilde{F} / \partial \tilde{U}, \tilde{B}=\partial \tilde{G} / \partial \tilde{U}$, and $\tilde{C}=\partial \tilde{H} / \partial \tilde{U}$ are Jacobian matrices; $I$ is a unit matrix; $\Lambda_{A}, \Lambda_{B}$ and $\Lambda_{C}$ are diagonal matrices whose diagonals are formed from the eigenvalues of 
the Jacobian matrices. The superscripts $(\cdot)^{+}$and $(\cdot)^{-}$denote vector splitting according to the Steger-Warming algorithm [11]. In Eqs. (27) and (28), the function "minmod" presents the limiter proposed by Jiang et al. [7,8]. In the general coordinate $(\xi, \eta, \zeta)$, the split forms of the convective flux vectors are simple and symmetrical, and are obtained through a series matrix operations. For the split flux vector of flux $\tilde{F}$ in the $\xi$ direction,

$$
\tilde{F}^{ \pm}=\frac{\rho}{2 J \bar{\gamma}}\left[\begin{array}{c}
C_{1}\left[2(\bar{\gamma}-1) \tilde{\lambda}_{1}^{ \pm}+\tilde{\lambda}_{n s+3}^{ \pm}+\tilde{\lambda}_{n s+4}^{ \pm}\right] \\
\vdots \\
C_{n s}\left[2(\bar{\gamma}-1) \tilde{\lambda}_{1}^{ \pm}+\tilde{\lambda}_{n s+3}^{ \pm}+\tilde{\lambda}_{n s+4}^{ \pm}\right] \\
u_{1}\left[2(\bar{\gamma}-1) \tilde{\lambda}_{1}^{ \pm}\right]+\left(u_{1}-k_{x} c\right) \tilde{\lambda}_{n s+3}^{ \pm} \\
+\left(u_{1}+k_{x} c\right) \tilde{\lambda}_{n s+4}^{ \pm} \\
u_{2}\left[2(\bar{\gamma}-1) \tilde{\lambda}_{1}^{ \pm}\right]+\left(u_{2}-k_{y} c\right) \tilde{\lambda}_{n s+3}^{ \pm} \\
+\left(u_{2}+k_{y} c\right) \tilde{\lambda}_{n s+4}^{ \pm} \\
u_{3}\left[2(\bar{\gamma}-1) \tilde{\lambda}_{1}^{ \pm}\right]+\left(u_{3}-k_{z} c\right) \tilde{\lambda}_{n s+3}^{ \pm} \\
+\left(u_{3}+k_{z} c\right) \tilde{\lambda}_{n s+4}^{ \pm} \\
2\left[(\bar{\gamma}-1) H-c^{2}\right] \tilde{\lambda}_{1}^{ \pm} \\
+(H-c \theta) \tilde{\lambda}_{n s+3}^{ \pm}+(H+c \theta) \tilde{\lambda}_{n s+4}^{ \pm}
\end{array}\right]
$$

where, $\tilde{\lambda}_{i}^{ \pm}=\frac{1}{2}\left(\tilde{\lambda}_{i} \pm \sqrt{\tilde{\lambda}_{i}^{2}+\epsilon}\right), i=1,2, \ldots, n s+4$; $\theta=k_{x} u_{1}+k_{y} u_{2}+k_{z} u_{3}, k_{x}=\xi_{x} / \Delta, k_{y}=\xi_{y} / \Delta, k_{z}=\xi_{z} / \Delta$, and $\Delta=\sqrt{\xi_{x}^{2}+\xi_{y}^{2}+\xi_{z}^{2}}, \xi_{x}, \xi_{y}, \xi_{z}$ are the transformation matrices between the physical and computational space, and $J$ is the transformation Jacobian. The split forms for $\tilde{G}$ and $\tilde{H}$ are similar to those of $\tilde{F}$.

An explicit and second-order Runge-Kutta integration method is used to advance the computational solution in time toward its steady state. The convergence criterion is defined as Res $=\frac{\left|\dot{m}_{\text {in }}-\dot{m}_{\text {out }}\right|}{\dot{m}_{\text {in }}} \leq 10^{-4}$, where $\dot{m}_{\text {in }}$ and $\dot{m}_{\text {out }}$ are the mass flow rates of some important species at the inflow and outflow boundaries of the nozzle, respectively, for example, helium for the validation case and oxygen for other cases. One run took about one month on a self-parallelized 64-bit workstation with four processors.

\subsection{Validation of the numerical simulations}

In order to verify numerical algorithms and validate numerical solutions, a cold flow with a transonic injection in a Research Assessment Device Improvement Chemical Laser (RADICL) slit nozzle was computed. The geometry used in the computation was chosen according to the experimental
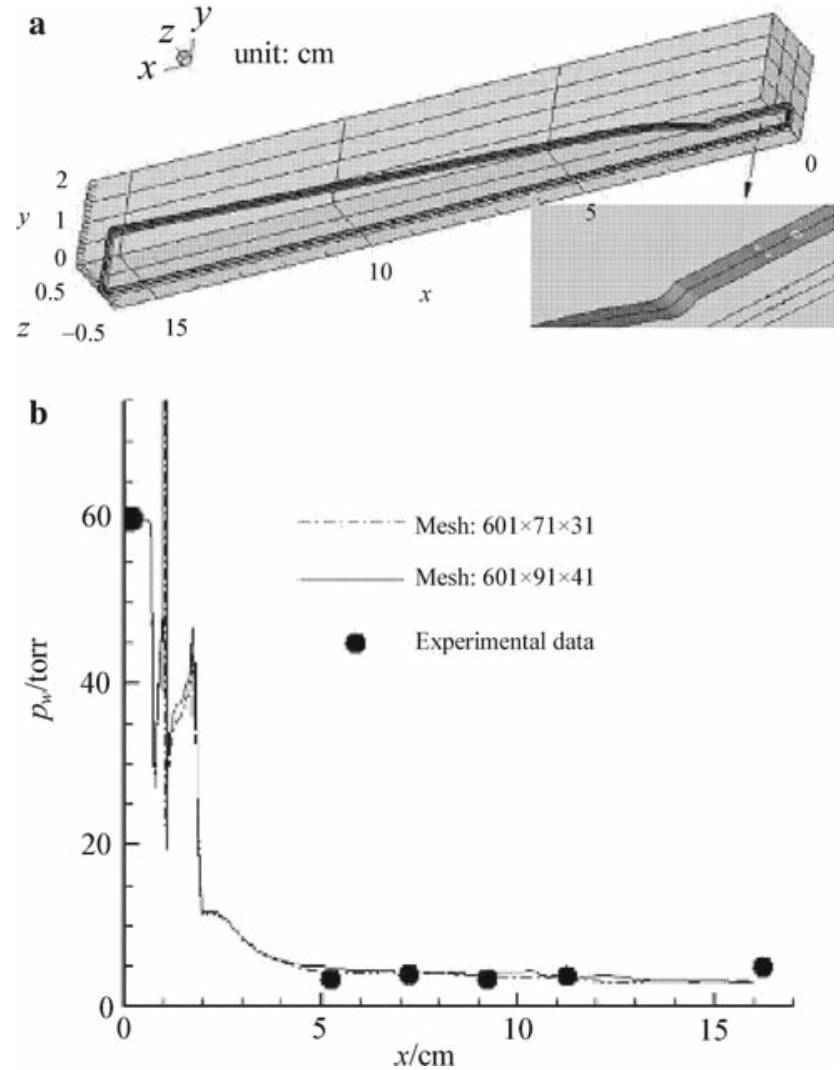

Fig. 2 RADICL slit nozzle flow simulation for validation and grid independence analysis: a computational domain and $\mathbf{b}$ comparison between the numerical result and experimental data in wall pressure

geometry used in [12]. The computational domain, which utilizes the unit cell approximation based on symmetry of the RADICL nozzle and the symmetry hypothesis of the multijet flows, is shown in Fig. 2a. A coarse grid $(601 \times 71 \times 31)$ and a fine grid $(601 \times 91 \times 41)$ were used to discretize the nozzle. The flow conditions were set as follows: (1) upstream from the nozzle throat, the second flow is injected into the primary flow perpendicularly through a big orifice with a diameter of $0.8128 \mathrm{~mm}$ and a small orifice with a diameter of $0.4064 \mathrm{~mm}$ in the unit cell; (2) helium is used for both the primary and second flows; and (3) the total temperature and pressure of the two flows are $T_{\mathrm{p}}=293 \mathrm{~K}, p_{\mathrm{p}}=7,955 \mathrm{~Pa}$, $T_{\mathrm{S}}=403 \mathrm{~K}$, and $p_{\mathrm{s}}=32,925 \mathrm{~Pa}$, where subscripts $\mathrm{p}$ and $\mathrm{s}$ denote the primary and second flows, respectively. For the boundary conditions in this calculation, the sonic inflow condition was set to the secondary injection, while the constant total pressure condition was imposed on the subsonic inflow condition for the primary flow.

Along with the measured experimental data, the computed wall pressure is shown in Fig. 2b. A good agreement between the experimental data and the numerical results in both the coarse and fine mesh has been achieved. The discrepancy in the pressure measurements is the highest near 
the downstream nozzle exit, which is considered to be a consequence of injecting a purge flow in the $z$ direction downstream of the nozzle throat in the experiment [12]. The application of the fine mesh shows only a minor change in the wall pressure. In the following simulations, the coarse mesh will be used. From the direct comparison of these results, it can be concluded that the numerical solutions are well validated for the frozen jet flow in the COIL, and the extension of the numerical algorithms to reactive flow simulations in the present study is acceptable.

\subsection{Flowfield and the small signal gain}

In the following computations, the computational domain and the injection schemes used are almost identical to those described in the validation section, except that the streamwise dimension of the nozzle is reduced to $60 \mathrm{~mm}$. A smaller domain allows the focusing of the near field of the jet mixing with a sufficient grid density at a reasonable computational cost. The mesh with $501 \times 71 \times 31$ grid nodes is used in all the subsequent numerical simulations. An exponential refinement algorithm is implemented to generate locally refined high density mesh near the jet orifices.

To analyze and compare the performance of jet mixing, the chemical reaction and signal gain generation in the mixing nozzle operating in three different cases that correspond to under-, full-, and over-penetration conditions have been studied. The primary flow conditions are identical for all computational cases. The premixed primary flow consists of $\mathrm{O}_{2}\left({ }^{3} \Sigma\right) / \mathrm{O}_{2}\left({ }^{1} \Delta\right) / \mathrm{H}_{2} \mathrm{O} / \mathrm{Cl}_{2} / \mathrm{He}$ with molar fractions of $1 / 0.69 / 0.16 / 0.24 / 5.77$. The total pressure, $p_{\mathrm{p} 0}=9.9 \mathrm{kPa}$, and the total temperature, $T_{\mathrm{p} 0}=315 \mathrm{~K}$, of the free stream are specified as the inflow boundary conditions of the nozzle intake. The secondary flow composing of $\mathrm{I}_{2} / \mathrm{He}$ (molar fraction 1:118.4) is injected perpendicularly to the primary flow at a sonic velocity in the subsonic section of the nozzle. While maintaining the temperature at $T_{\mathrm{s} 0}=415 \mathrm{~K}$, three different jet pressures, $p_{\mathrm{s} 0}=16.1,20$ and $24 \mathrm{kPa}$, corresponding to the under-, full- and over-penetration conditions of the transverse jet in the nozzle flow, are used. It is well known that the round-jet/cross-stream interaction produces a complicated flowfield that depends primarily on the effective velocity ratio or blowing ratio, $r=\sqrt{\rho_{\mathrm{s}} u_{\mathrm{s}} / \rho_{\mathrm{p}} u_{\mathrm{p}}}$. Therefore, the jet pressure values represent the different jet densities and, in turn, the different effective velocity ratios considered in the simulations.

A second jet, when issued into a crossflow, will be bent to the streamwise direction of the primary flow. Generally, the fluid is entrained and the vorticity in both the jet and the cross flow stretches and aligns to produce four dominant flow structures: the jet shear layer, the counter-rotating vortex pair (CVP) embedded in the jet trajectory, the horseshoe vortices, and the wake structures. These structures and vortices are
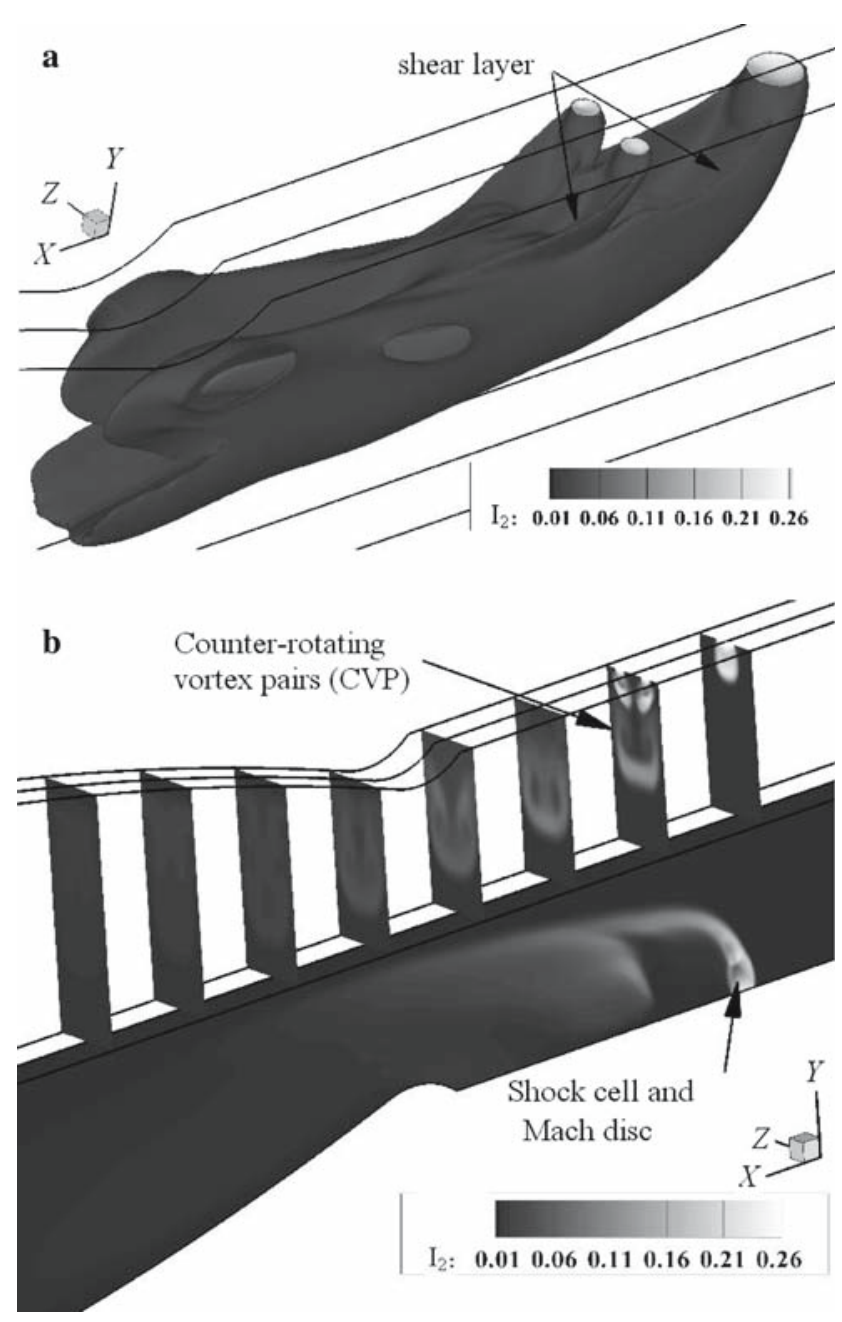

Fig. 3 Density of molecular iodine $\mathrm{I}_{2}$, in the near field of the transverse jet (under-penetration). a 3D contour and $\mathbf{b}$ profiles in lateral planes (upper half) and longitudinally symmetric plane (lower) (normalized by the primary flow density)

helpful to enhance the mixing process, and then chemical reactions. The jet structures, production of laser medium, and small signal gain fields will be analyzed contrastively in the following paragraphs.

In Fig. 3, the complex flow structures in the near flowfield of the transverse jet are demonstrated with the distribution of molecular iodine. The horseshoe-shaped structures associated with the transverse jet flow can be seen clearly in Fig. 3a and $b$ due to the effect of shear stress on the jet fluid as it penetrates into the primary flow. The shear layer and counter-rotating vortex pair are induced by the stretching and rolling of the interface between the primary and jet flows. All these flow structures and processes increase the entraining surface area, as well as the concentration gradients, and in turn the diffusion. The under-expanded jet condition induces the shock cell and Mach disc structure near the jet orifice, as shown in the lower half of Fig. 3b. The density of the 


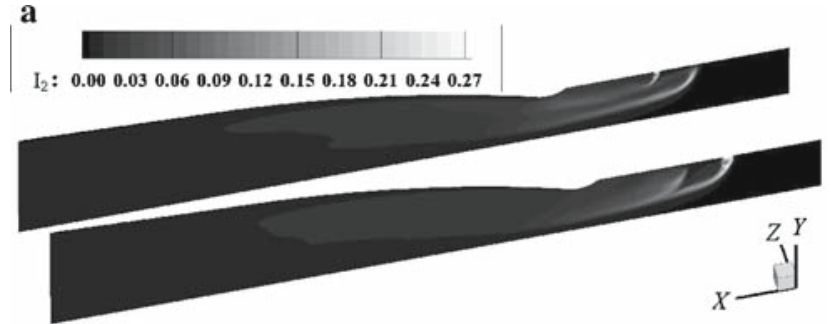

b

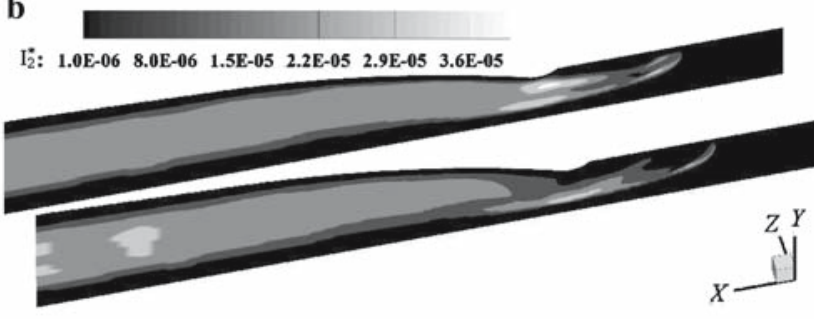

c

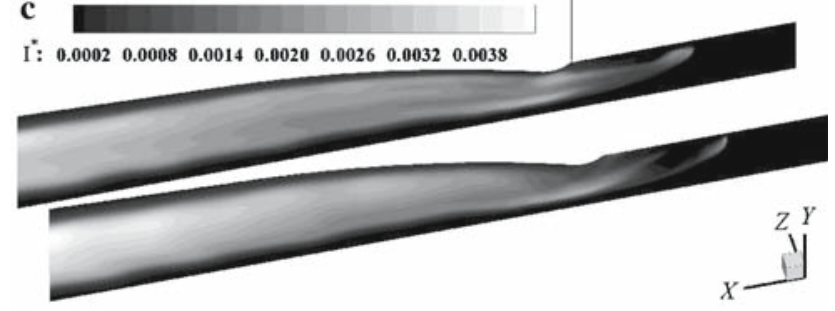

d

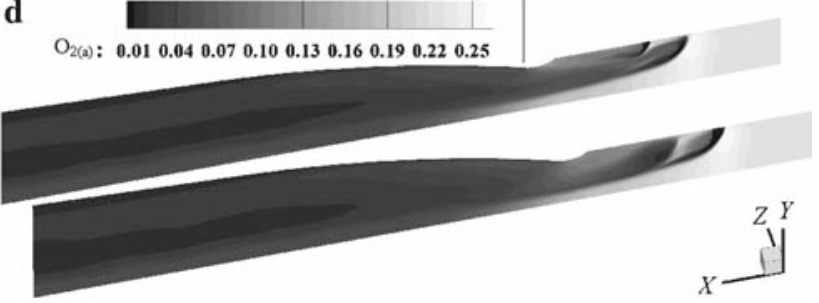

Fig. 4 Distribution of each species in the symmetric planes of the smaller (upper) and larger (lower) orifices (normalized by the primary flow density)

molecular iodine decreases quickly as a result of the expansion and dissociation in the supersonic region of the mixing nozzle, as shown in Fig. 4a. The presence of intermediate products, $\mathrm{I}_{2}^{*}$, as shown in Fig. $4 \mathrm{~b}$, highlights the chain dissociation mechanism of the molecular iodine mentioned in Fig. 1. The laser medium field is homogeneous throughout most of the region downstream from the nozzle throat, except in the boundary layer and the core section of the nozzle flow. Owing to the under-penetration condition, the singlet oxygen molecules are not utilized efficiently, as shown in Fig. $4 \mathrm{c}$ and d. The two jets from the small orifices increase the spatial distribution of the iodine in the field where the former jet cannot reach, as given in Figs. 3 and 4a.

The small signal gain that determines the laser beam output is proportional to the concentration difference $\left(\left[\mathrm{I}^{*}\right]-\right.$ 0.5[I]). In Fig. 5a, the ground state atomic iodine near the nozzle throat is produced at a relative high rate due to the
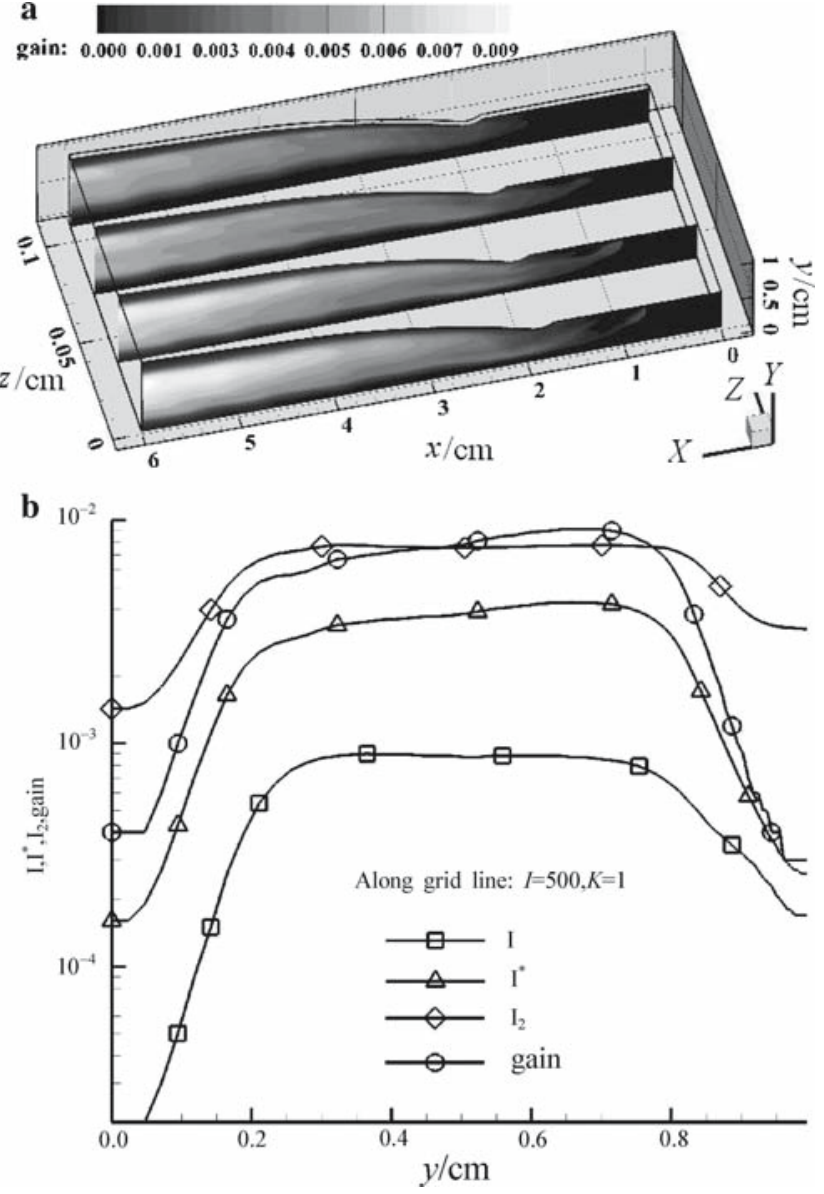

Fig. 5 Small signal gain and related species (under-penetration): a profiles of gain in longitudinal planes and $\mathbf{b}$ spanwise curves at the nozzle exit for $\mathrm{I} \mathrm{I}^{*}, \mathrm{I}_{2}$ (normalized by the primary flow density) and gain $\left(\mathrm{cm}^{-1}\right)$

rapid dissociation of $\mathrm{I}_{2}$ and the relaxation of the pumping reaction, which results in a relative low gain value. This coupled process of gas dynamics and chemical kinetics makes the flowfield more complex. There are two regions of the low gain, as seen in Fig. 5a and $\mathrm{b}$ in the boundary layer and near the symmetric plane of the nozzle. A proper penetration condition should be applied to produce a better gain field and the more efficient utilization of the energy carrier, $\mathrm{O}_{2}\left({ }^{1} \Delta\right)$. The jet trajectory reaches the horizontal symmetric plane, i.e. the full penetration condition is achieved, when the jet pressure is set to $20 \mathrm{kPa}$. The small signal gain and some key species are shown in Fig. 6. One of the problems, the trapezium profile as shown in Fig. 5b, associated with underpenetration is overcome by the full penetration jet mixing as seen in Fig. 6a. The effective gain region extends to most of the spanwise range of the nozzle, except in the boundary layer. In Fig. 6b, the different evolutions of the ground state (denoted by squares) and the pumped (denoted by triangles) iodine in the transonic region of the nozzle indicates the coupling between the chemical reaction mechanism and 

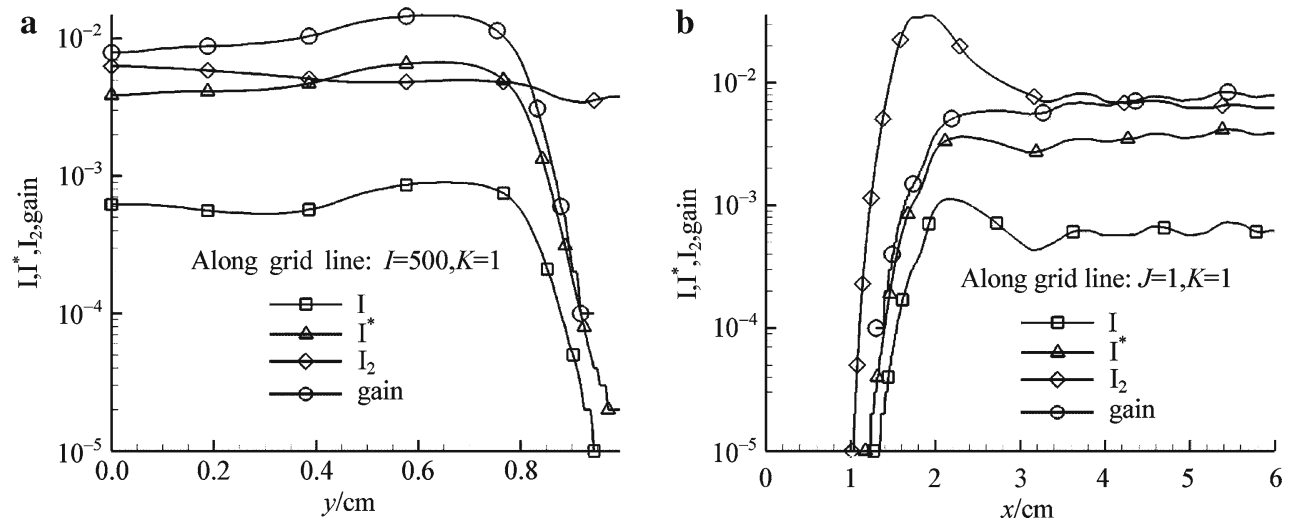

Fig. 6 Species variation curves for $I, I^{*}, I_{2}$ (normalized by the primary flow density) and gain $\left(\mathrm{cm}^{-1}\right)$ in the full penetration case: a spanwise variation at the nozzle exit and $\mathbf{b}$ streamwise variation in the longitudinal symmetric plane $(x$-throat $=1.888 \mathrm{~cm})$

the gas dynamics. Both non-dimensional densities of these two species decrease abruptly due to the supersonic expansion downstream from the nozzle throat $(x=1.188 \mathrm{~cm})$. The excited iodine undergoes a smaller drop than the ground state atomic iodine and then increases gradually, which shows that the generation of $I^{*}$ via an exothermal pumping reaction benefits more from the flow cooling process than from the rarefication associated with the supersonic expansion of the nozzle flow. Consequently, the different variation trends of these two species accelerate the growth of the small signal gain.

Three parameters, the utilization of the singlet oxygen, the dissociation ratio of the molecular iodine, and the pumping ratio of atomic iodine, are generally used to evaluate the performance of the mixing nozzle. These parameters demonstrate 3D features according to the different $z$-planes, as shown in Fig. 7. These 3D features imply that the optimum spatial distribution of the chemical performance cannot be obtained, even if the optimum jet penetration condition is achieved. Therefore, a trade-off exists between the jet dynamic and the kinetic process, and it needs further study and optimization in the future.

In the case of over-penetration, the jet pressure is increased to $p_{\mathrm{s}}=24 \mathrm{kPa}$. The numerical results show that strong unsteady structures are induced by the impingement between the jets. Bubbles containing singlet oxygen molecules and iodine molecules can be found in Fig. 8a and b, respectively. Such unsteadiness and heterogeneousness in the jet mixing region will directly translate into unsteadiness and heterogeneousness in the laser gain, and finally result in the time dependency of the laser power output [2]. Moreover, the heterogeneousness in the laser cavity also leads to the degeneration of the laser beam quality due to the asymmetrical refractive index of the fluid mixture. The over-penetration impingement also causes higher pressure losses. For these reasons, the over-penetration jet is not recommended in the COIL mixing nozzle design.

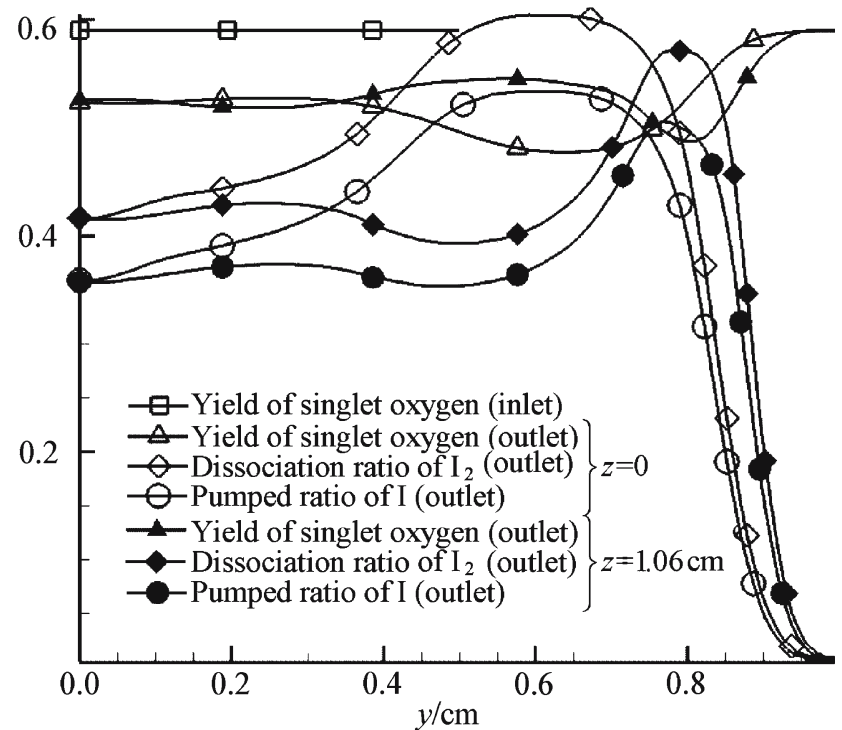

Fig. 7 Spatial variation of the yield of $\mathrm{O}_{2}\left({ }^{1} \Delta\right)$, dissociation ratio of $\mathrm{I}_{2}$ and the pumping ratio of I: open symbols, $z=0$ and filled symbols, $z=1.06 \mathrm{~mm}$

\section{Conclusions}

Various numerical simulations of 3D chemically reacting flow have been performed to obtain further understanding on the physical and chemical processes underlying the nozzle flow of a chemical oxygen-iodine laser. Several operation conditions with different transverse jet pressures have been studied corresponding to the different conditions. The flow structures associated with transverse jets in cross-flow are well captured and seem to play an important role in the mixing of flows in the COIL nozzles. In an under-penetration jet, the singlet oxygen molecules in the core flow are not effective in transferring energy to the laser mediums and will be discharged with the exhaust gases. Such the energy waste will result in a lower chemical efficiency. In over-penetration 

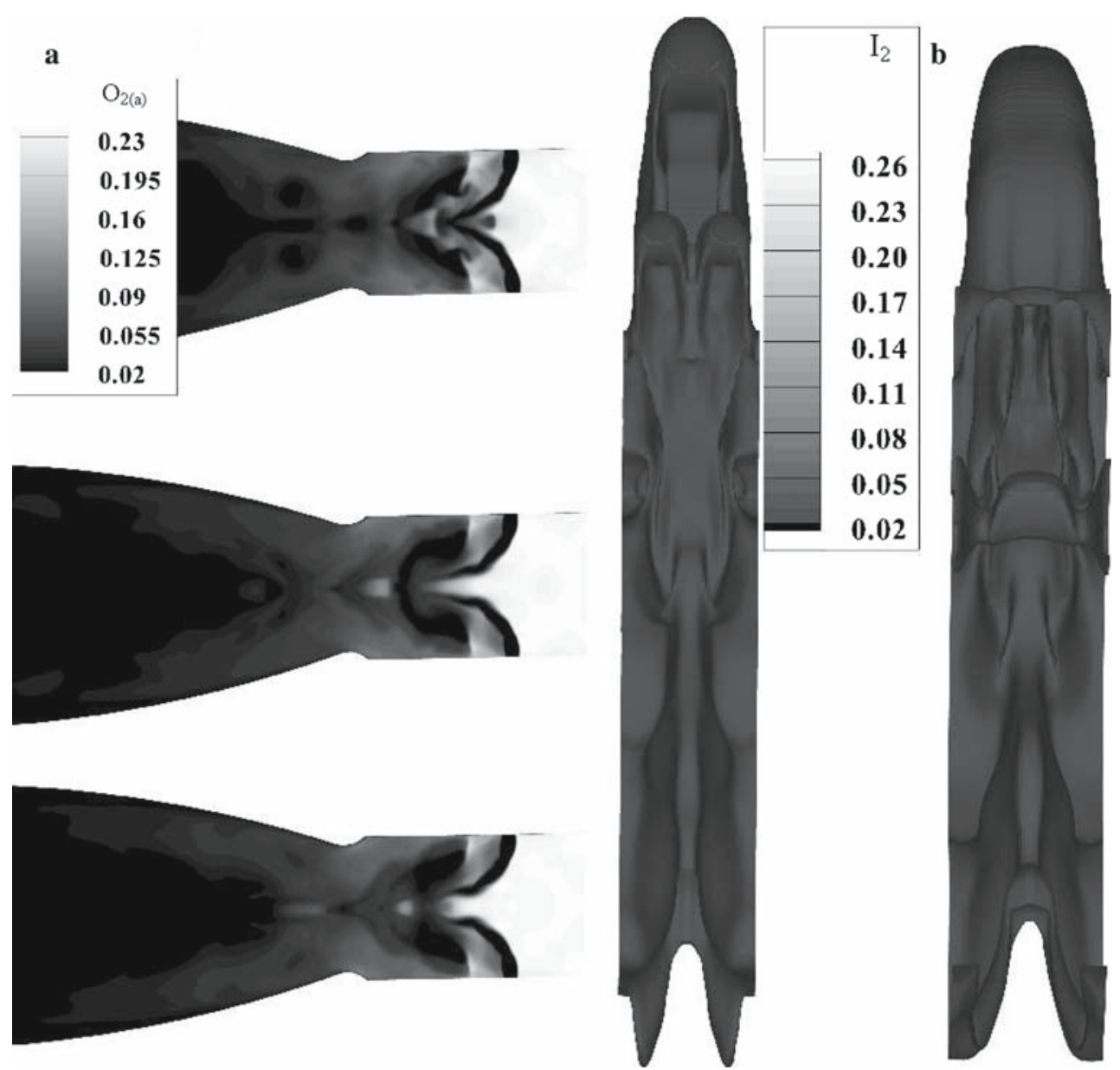

Fig. 8 Unsteady structures and impingement (near field in over-penetration): a instantaneous distribution of $\mathrm{O}_{2}\left({ }^{1} \Delta\right)$, in the $z=0$ plane (time interval is 4.5) and $\mathbf{b}$ top and bottom views of the $3 \mathrm{D}$ contour of $\mathrm{I}_{2}$ (normalized by the primary flow density)

condition, it is expected that the unsteady and heterogeneous structures induced by the impingement will translate into the degeneration of the laser beam quality. Under a full penetration jet condition, a relatively uniform flowfield and gain field can be achieved. It should be noted that even in such the condition, the chemical reaction field, to some extent, has some non-uniformities and 3D features. Further studies are needed in the future.

Acknowledgments The authors would like to thank Prof. B.G. Wu and Prof. G.W. Yang at the Institute of Mechanics, and Prof. F.T. Sang, F. Chen and Dr. B.J. Fang at the Dalian Institute of Chemical Physics of Chinese Academy of Sciences. This work was partially supported by the Korea Research Foundation Grant No. KRF-2005-005-J09901.

\section{References}

1. Bohn, W.L.: International COIL technology survey. In: Abramski, K.M., Plinski, E.F., Wolinski, W. (eds.) Proceeding of SPIE, vol. 5120, pp. 301-307. Bellingham, WA (2003)
2. Madde, T.J.: Aspects of 3-D chemical oxygen-iodine laser simulation. In: Abramski, K.M., Plinski, E.F., Wolinski, W. (eds.) Proceeding of SPIE, vol. 5120, pp. 363-375, Bellingham, WA (2003)

3. Hu, Z.M., Sun, Y.Y., Wu, B.G., Jiang, Z.L.: Numerical simulation on the flow field of COIL mixing nozzle with injection from subsonic region. High Power Laser Particle Beams 17(4), 481484 (2005)

4. Mikatarian, R.R., Jumper, E.J., Woolhiser, C.C.: Fluid dynamic issues in continuous-wave, short-wavelength chemical laser. AIAA Paper, pp. 88-2748 (1988)

5. Miller, J.A., Jumper, E.J.: Role of mixing in the chemical oxygeniodine laser reactions. AIAA J. 32(6), 1228-1233 (1994)

6. Paschkewitz, J., Shang, J., Madden, T.J.: An assessment of COIL physical property and chemical kinetic modelling methodologies. AIAA Paper, pp. 2000-2574 (2000)

7. Eppard, W.M., McGrory, W.D., Godfrey, A.G., Cliff, E.M., Borggaard, J.T.: Recent advances in numerical techniques for the design and analysis of COIL system. AIAA Paper, pp. 2000-2576 (2000)

8. Jiang, Z.L., Takayam, K., Chen, Y.S.: Dispersion conditions for non-oscillatory shock capturing schemes and its applications. Computat. Fluid Dyn. J. 4, 137-150 (1995)

9. Jiang, Z.L.: On dispersion-controlled principles for non-oscillatory shock-capturing schemes. Acta Mech. Sin. 20(1), 1-15 (2004) 
10. Hu, Z.M., Gao, Y.L., Zhang, D.L., Yang, G.W., Jiang, Z.L.: Numerical simulation of gaseous detonation reflection over wedges with a detailed chemical reaction model. Acta Mech. Sin. 36(4), 385-392 (2004) (in Chinese)

11. Steger, J.L., Warming, R.F.: Flux vector splitting of the inviscid gas dynamic equations with applications to finite difference method. J. Computat. Phys. 40, 263-293 (1981)
12. Miller, J.H., Shang, J.S., Tomaro, R.F., Strang, W.Z.: Computational of COIL nozzle flowfields with transonic injection. AIAA Paper, pp. 2000-2575 (2000) 